\title{
Uncommon Location of Lipoma: Extraluminally Vagina
}

\author{
Baris Guner', Nevzat Saat ${ }^{1} \&$ Mustafa Usta ${ }^{2}$
}

\begin{abstract}
Background: Lipomas are defined as a soft mass of well-differentiated adipose cells among mesenchymal tumors. Considering the localization of lipomas, these masses are commonly seen in subcutaneous tissue. The female reproductive tract is a rare site for development of lipomas and clinical findings appear depending on the size and localization. The connection of the mass with the vagina lumen causes different clinical findings. Intraluminal tumors protrude and arise from the vulva, while extraluminal tumors lead to perineal swelling. This case describes clinical, ultrasonographic, histological examination, and surgical management of extraluminal vaginal lipoma.

Case: The present study reports a case of extraluminal vaginal lipoma in a 10-year-old Pitbull crossbred presented with anamnesis of a protruded tissue from the vulvar lips and dysuria following 6-month swelling in the perineum. Clinical examination revealed that the swelling was obvious and localized mostly on the lower left side of the perineum and the vaginal mucosa was protruded from the vulvar lips due to a mass. By vaginal palpation, the mass was not associated with the vaginal mucosa. The mass was located in the perivaginal region and transvaginal ultrasonography revealed a hypoechogenic mass. Based on clinical and ultrasonographic findings, surgery was recommended. The mass was adherent to the serosa of the vagina and it did not enclose the vaginal mucosa. Excision of mass was performed with careful blunt dissection avoiding any urethral disruption and periurethral tissues. After the mass was determined to be extraluminal, there was no need for excision of the vaginal tissue with the mass during operation. Using histopathological examination the mass was identified as a lipoma composed of adipocytes. In addition, ovariohysterectomy was not recommended following the diagnosis of vaginal lipoma.

Discussion: Lipomas uncommonly can be localized in unpredicted rare areas such as vagina and vulva in older bitches. Although the majority of reported benign tumors in dogs recommend the surgery, medical treatment including the steroid injections is the other option to limit the lipomas. Considering the vaginal tumors, the surgical approach is also planned as laparotomy since leiomyomas are more common than lipomas. This approach partially results from the adherence of tumors to vaginal tissue. In addition, the location of the mass together without the adjacent tissue damage depending on whether it is intraluminal or extraluminal would make complete tissue removal. In previous reports, while symptoms such as perineal hernia of the lipoma were emphasized, the connection of the lipoma with the vaginal lumen was not evaluated as a significant factor determining the surgical approach. The mass was not connected to the vaginal mucosa and it was determined as extraluminal lipoma in the present study. Mass excision was performed by blunt dissection, avoiding urethral disruption and periurethral tissues. Unlike the most common approach in leiomyomas, this report did not require a total vaginectomy to remove the mass from the vagina. The ovariohysterectomy was not needed to be performed in the present study as in intraluminal vaginal tumors. It was believed that adhesions of lipoma with vaginal lumen is a determining factor in the combination of surgical techniques such as episiotomy and/or ovariohysterectomy. Lipomas should be considered among the tumors in the vaginal region of dogs brought with the complaint of swelling in perineum and protrusion of vaginal tissue.
\end{abstract}

Keywords: genital tumors, lipoma, vagina, dog. 


\section{INTRODUCTION}

Genital tumors comprise approximately 3\% of all tumors and they are the second most common tumors after mammary gland tumors in dogs [11]. Tumors in the female reproductive tract are not recognized at an early stage and are generally benign [7]. Lipomas are benign tumors composed of adipocytes less commonly seen among mesenchymal tumors in the female reproductive tract [3,5], which are common benign tumor types reported in about $16 \%$ of dogs but are rarely detected in other species [1,5]. This benign tumor frequently occur in the subcutaneous tissues and are one of the three most common types of cutaneous tumors with $7.1 \%$ percentage and can be localised in unpredicted rare areas such as thoracic cavity, abdominal cavity, spinal cavity, thymus, epiglottis, trachea, vagina and vulva [3]. There has been very limited information about vaginal lipoma in dogs after examination of original sources [1,3] and previous reports $[4,8]$.

\section{CASE}

A 10-year-old, Pitbull crossbred bitch weighing $23.4 \mathrm{~kg}$ was brought to the University Clinics of Balıkesir University with the complaint of a perineal swelling and dysuria (Figure 1). According to anamnesis, she gave birth 6 years ago, showed oestrus at approximately 7-month intervals and was not neutered. A swelling of approximately $1 \mathrm{~cm}$ in diameter was noticed around the perineal region when last proestrus bleeding was observed 6 months ago. The swelling gradually increased and difficulty in urination began to develop.

In the clinical examination; physical examination parameters such as pink mucous membrane, body temperature $\left(38.7^{\circ} \mathrm{C}\right)$, capillary refill time $(2 \mathrm{~s})$ were within the normal range. Increased respiratory rate (56 breaths per minute) and heart rate (128 bpm) were assumed to be caused by its excitement. The data for hematological parameters were determined by using hematology analyzer (Abacus Junior Vet5) ${ }^{1}$. Hemoglobin concentration was higher than the reference interval in the present case. Other blood parameters were revealed within the reference range. The swelling was obvious and localized mostly on the lower left side of the perineum. It was found that vaginal tissue protruded from the vulva with a diameter about 2.0 to $2.5 \mathrm{~cm}$. There was mucosal ulceration on a large area of the vestibular vagina that protruded (Figure 1). The mass was palpable minimally throughout the vagina following the rejection of the vestibular vagina from the vulvar lips. It was revealed that the mass prevented the urination partially and mass was not connected with the vaginal lumen. Transvaginal ultrasonographic examination (Ibex) ${ }^{2}$ was performed to determine the characterization of mass. The mass was observed as encapsulated and hypoechogenic (Figure 2). Although the dimension of the mass was not revealed clearly, the diameter of the mass was calculated at least $65 \times 44$ $\mathrm{mm}$ with the transvaginal ultrasonographic examination. An anechogenic, fluid-filled urinary bladder did not protrude with mass from vulvar lips and it retained the anatomical position. In addition, no abnormalities such as cystic endometrial hyperplasia and ovarian cyst were found in genital tract during the abdominal ultrasonography. It was decided to remove the mass surgically based on anamnesis, clinical and ultrasonographical findings.

The perineum was prepared aseptically. Premedication was performed with atropine sulfate [0.04 $\mathrm{mg} / \mathrm{kg}$ b.w., subcutaneously $]^{3}$ and xylazine $\mathrm{HCl}$ [1.5 $\mathrm{mg} / \mathrm{kg}$ b.w., intramuscularly $]^{4}$. Meloxicam [0.2 mg/ kg b.w., subcutaneously, every 24 h for 3 days] $]^{5}$ was administered for postoperative analgesia. The general anesthesia was induced with ketamine $\mathrm{HCl}[6.0 \mathrm{mg} / \mathrm{kg}$ b.w., intramuscularly $]^{6}$ and maintained with ketamine $\mathrm{HCl}$ and diazepam ${ }^{7}$ combination with the proportion of $1 / 2$. Amoxicillin-clavulanic acid $[20 \mathrm{mg} / \mathrm{kg}$ subcutaneously $]^{8}$ was administered every $12 \mathrm{~h}$ for 5 days. The bitch was placed in sternal recumbency. After urethral catheterization with a foley catheter, an incision was performed ventrolaterally at the left vulvar lip. The urine flow was continuously controlled during the operation and the mass was isolated. The smooth mass was adherent to the serosa of the vagina and it did not enclose the vaginal mucosa (Figure 3A-B). Excision of mass was performed with careful blunt dissection avoiding any urethral disruption and periurethral tissues. It did not require total vaginectomy to remove the mass from the vagina. Closure of the excision gap in the perivaginal region was performed with $2 / 0$ absorbable suture $^{9}$. Reconstruction of the anatomic appearance of the vagina and vulva was performed (Figure 4)

Mass was fixed in formalin $10 \%$ and submitted for histopathological evaluation. Excised ovoid tumoral mass sized $3 \times 8 \times 12 \mathrm{~cm}$ and had a smooth surface with no capsulation. The resected mass weighed $220 \mathrm{~g}$. On the cut surface, the tumoral mass was white in color 
showing occasional pink to tan colored indentations or irregular areas (Figure 3B). The tumoral mass was soft in consistency and compatible with adipose tissue. The smaller pieces of the tumoral mass were seen to float in formalin solution. Formalin fixed paraffin embedded tissue samples were cut at $5 \mu \mathrm{m}$ and stained with $\mathrm{H} \& \mathrm{E}$ and Masson trichrome for histopathological examination. Microscopically, the sections were observed as characteristic lipoma, which was composed of mature adipocytes. Tumor cells, which are non polymorphic,

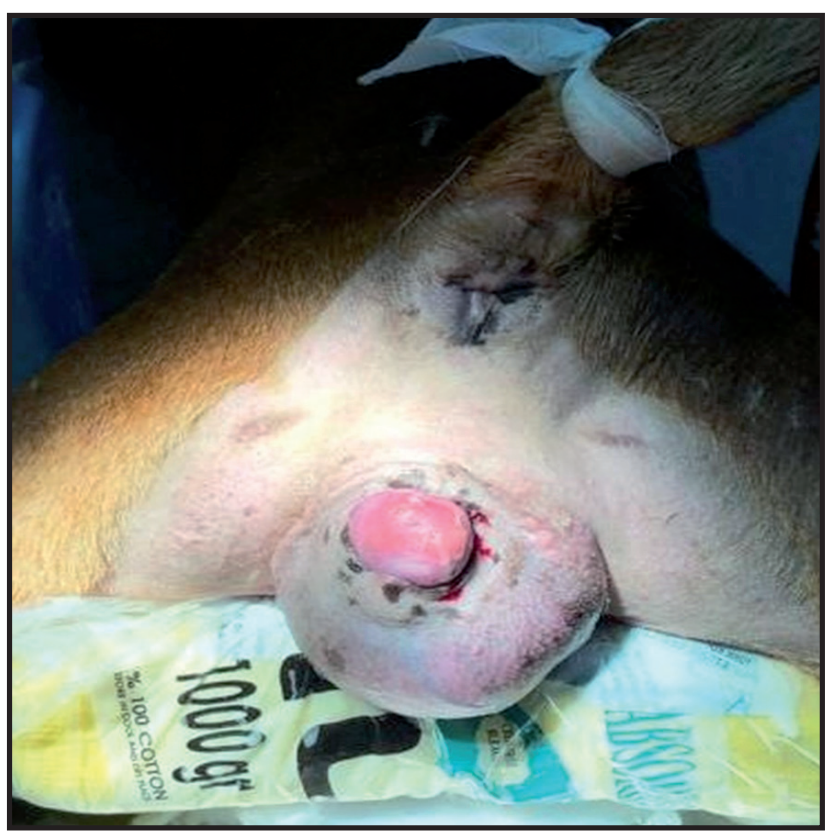

Figure 1. A 10-year-old pitbull crossbreed dog shows swelling in the perineum caused by vaginal lipoma before the surgical management.

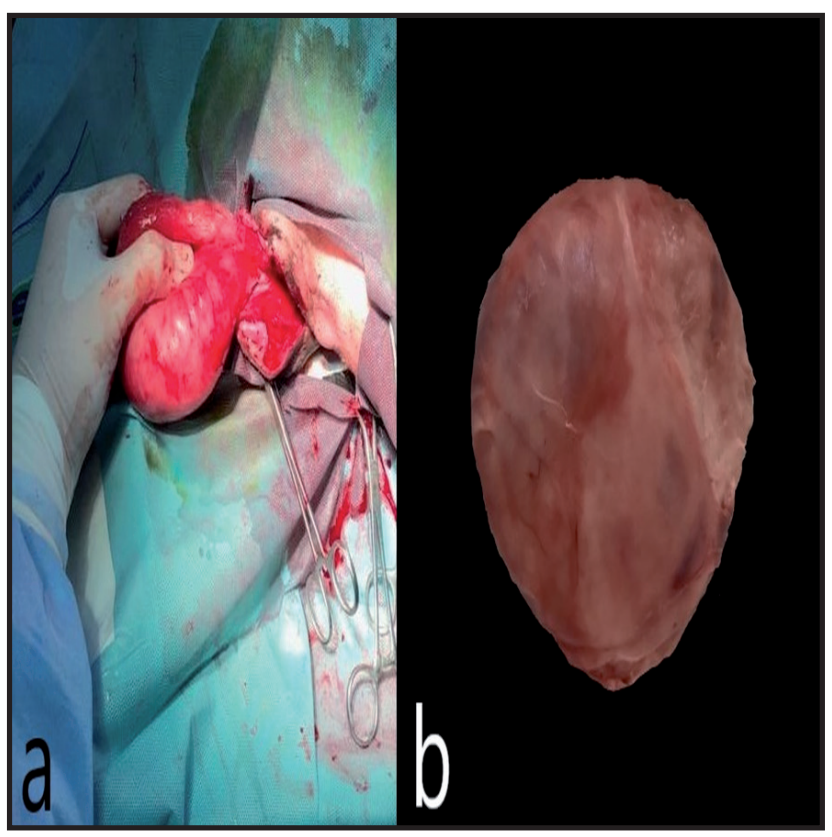

Figure 3. A- Intraoperative view of mass without adhesion to vaginal mucosa following blunt dissection. B- Cut surface of the tumoral mass after resection. had a large vacuole covering the entire cytoplasm pushing the nucleus to the periphery. Cells were supported by fine fibrous tissue septa and blood vessels (Figure 5A-B). Necrosis and atypical cells were not observed.

\section{DISCUSSION}

Lipomas are defined as a soft mass of well-differentiated adipose cells among mesenchymal tumors. Leiomyomas, fibroleiomyomas and fibromas are common, while leiomyosarcomas, lipomas, mastocytomas,

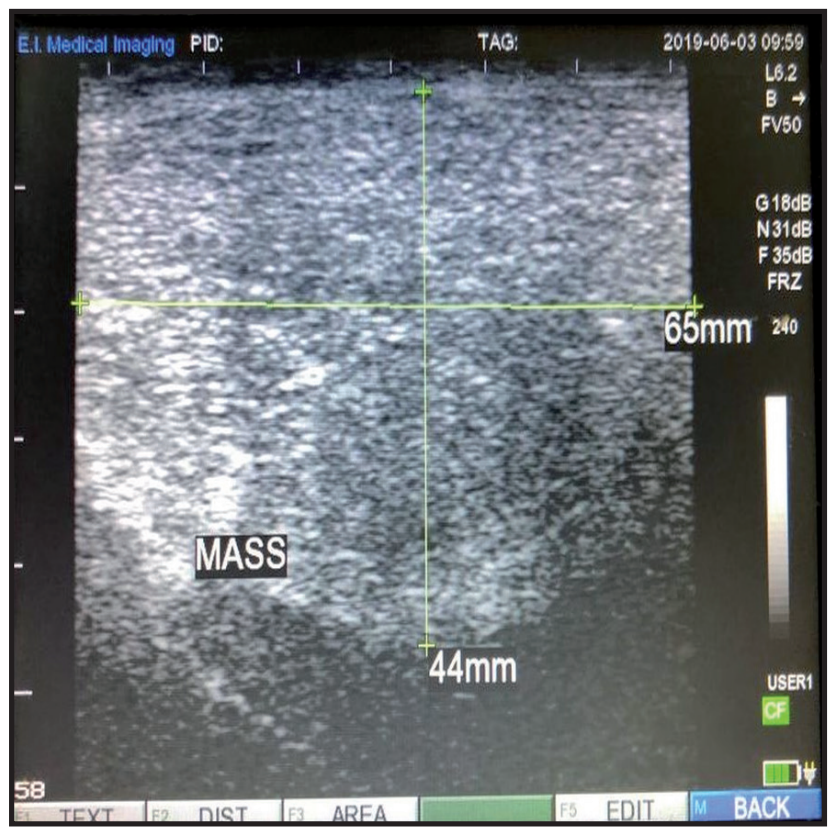

Figure 2. The transvaginal ultrasound apperance of mass with encapsulated hypoechogenic.

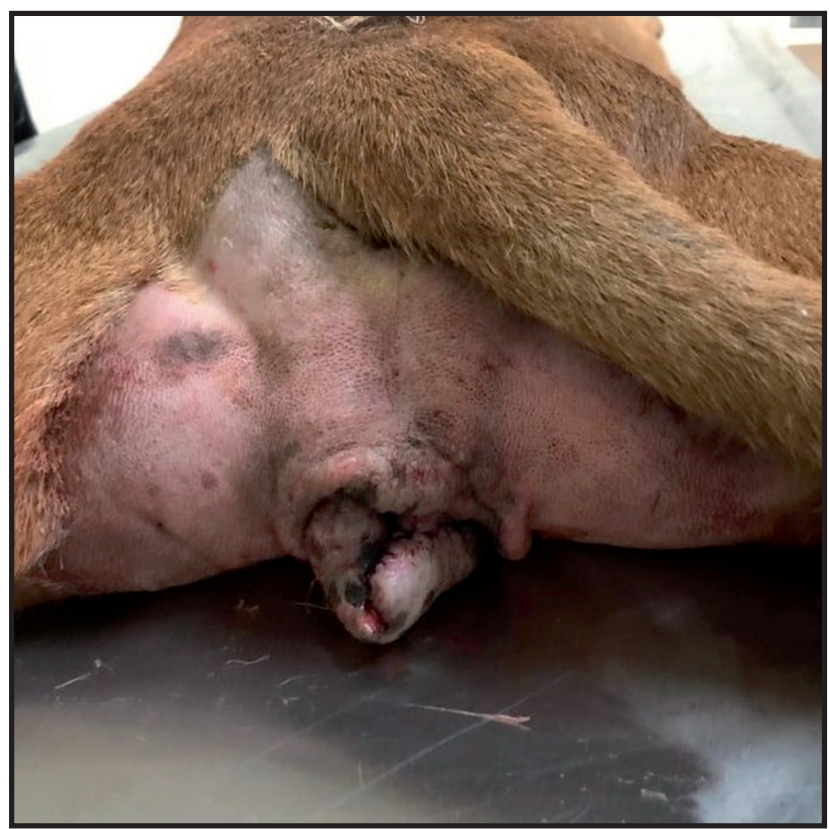

Figure 4. Reconstruction of the anatomic appearance of the vagina and vulva was performed after surgery. 


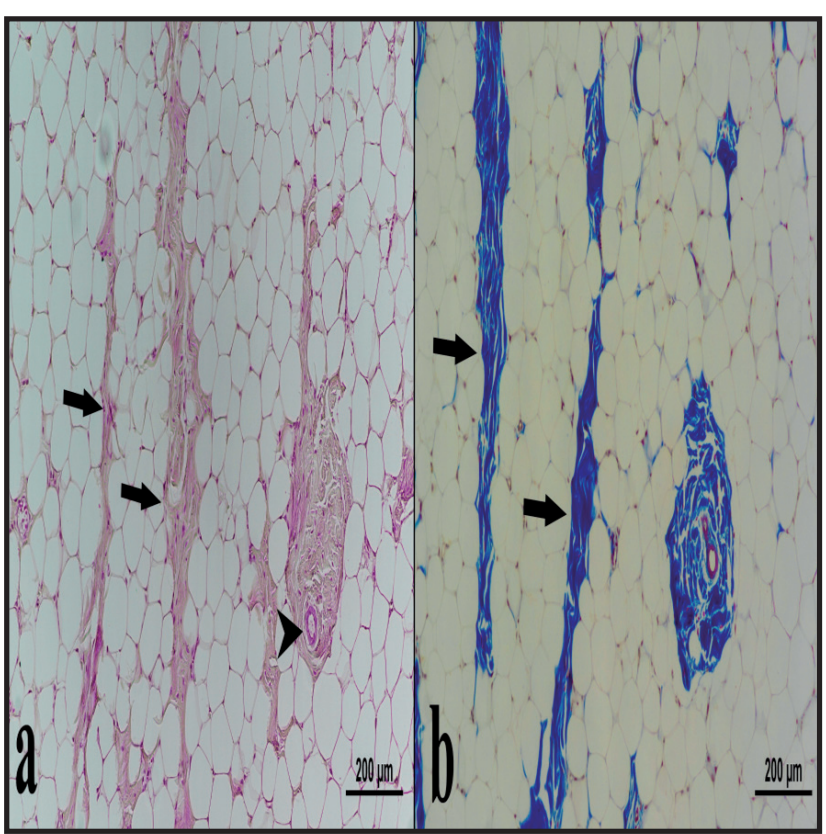

Figure 5. Histopathological view of the tumoral mass. A- Tumor cells having a large vacuole covering the entire cytoplasm. Tumor cells were supported by fine mesenchymal tissue (arrow) and blood vessels (arrow head) $[\mathrm{H} \& \mathrm{E}]$. B- Mesenchymal tissue of the tumor was formed by fibrous tissue septa (arrow) [Masson trichrome].

adenocarcinomas and squamous cell carcinomas are rarely seen among tumors of mesenchymal origin [11]. In terms of the location of the tumors in the female reproductive system, it was determined 8 times more in the vagina and vulva compared to those in the uterus [12]. Vaginal lipomas have been found to occur sporadically with a low incidence in older bitches [3]. Furthermore, $65-97.5 \%$ of dogs with vaginal and vulvar tumors were not neutered. In the present report, age and reproductive status of bitch were consistent with the previous studies of genital tumors in bitch $[4,8]$. There has been weak evidence related to development of lipomas except for predisposition such as genetic and breed [10]. There has been a limited number of reports studying vaginal lipoma in dogs $[4,8]$.

The mass was not connected to the mucosa and associated with serosa of vaginal tissue, so it was located as extraluminal. The mass was not connected to the mucosa and associated with serosa of vaginal tissue, so it was located as extraluminal in the present case. The connection of lipoma with a vaginal lumen was not emphasized by previous reports that it is a significant factor determining the clinical symptoms $[4,8]$ which may vary depending on the localization and size of the mass. The connection of the mass with vaginal lumen causes the mass protruding through the vulva but extraluminal tumors cause the enlargement of the localized area and perineal swelling [2]. Besides, extraluminal location of tumors was reported to cause tenesmus and constipation [2]. However, these findings were not observed and extraluminal mass caused dysuria due to the pressure of the vaginal tissue on the urethral tract. As in the previous case evaluating vaginal lipoma [8], the mass did not cause perineal hernia in pelvic diaphragm failure in the present case.

Steroid injection is effective to regress by $70 \%$ for the small lipomas in dogs. However, the mechanism for local steroid injection has not been known and this regression is not an exact solution after 10 months [5].

The most commonly reported vaginal tumors are leiomyomas which is reported to be located intraluminally. Surgical resection with blunt dissection was performed for lipomas disrupting physiological function of organs $[2,10,12]$. Furthermore, the mass excision with episiotomy or/and ovariohysterectomy is needed to be performed for leiomyomas commonly located as intraluminal [3,9]. In addition, ovariohysterectomy is necessary for the role of estrogens in etiology of leiomyomas [2]. The mass was not attached to the vaginal mucosa and was determined to be extraluminal. Therefore, ovariohysterectomy or/and vaginectomy was performed for neither etiology nor surgical approach in the present study.

The characterization of the vaginal mass via histopathological evaluation needs to be detected in terms of malignancy and prognosis [3]. In lipomas, tumoral cells of slightly larger adipocytes slightly differ from the surrounding adipose tissue [6]. Fat cells and mesenchymal elements such as smooth muscle cells and fibrous tissue cells are found together in the tumoral mass of the adipose tissues, they may rarely form tumor mass alone $[1,3,6]$. Masson trichrome stained sections of the mass were examined for the presence of smooth muscle and fibrous tissue cells, and shown to be supported by septa consisting of fibrous tissue cells. In the present case the histopathological observations of mass were also in accordance with the literature [1].

In conclusion, histopathology plays an important role in determining the prognosis and characterization of vaginal masses causing nonspecific clinical findings. The connection of the lipoma in the perivaginal region to the vaginal lumen is a determinant factor for the clinical findings and the combination of surgical techniques such as episiotomy and/or ovariohysterectomy in addition to excision of the lipoma. The patient owner had no complaints about dysuria and swelling around the perineum after operation in the present case. 


\section{MANUFACTURERS}

${ }^{1}$ Diatron Group. Vienna, Austria.

${ }^{2}$ E.I. Medical Imaging. Loveland, CO, USA.

${ }^{3}$ Vetaş. Ankara, Turkey.

${ }^{4}$ Ata Fen Veterinary Products. Izmir, Turkey.

${ }^{5}$ Hektaş Ticaret T.A.Ş. Kocaeli, Turkey.

${ }^{6}$ Richer Pharma A.G. Wels, Austria.

${ }^{7}$ Deva Holding A.S. Kocaeli, Turkey.
${ }^{8}$ Pfizer Türkiye. İstanbul, Turkey.

${ }^{9}$ Çetin Kimya Ltd Şti. Adana, Turkey.

Acknowledgements. We thank Prof. Dr. Musa Karaman for their help with contributing to the manuscript.

Declaration of interest. The authors have declared no conflict of interests. The authors alone are responsible for the content and writing of the paper.

\section{REFERENCES}

1 Agnew D. \& MacLachlan N. 2016. Tumors of the genital systems. In: Meuten D.J. (Ed). Tumors in Domestic Animals. 5th edn. Ames: John Wiley \& Sons, pp.689-722.

2 Bodinga H.A., Abubakar N., Abdullahi N. \& Hassan T. 2019. Vaginal Leiomyoma in a Crossbred Rottweiler Female Dog - A Case Report. Asian Journal of Research in Animal and Veterinary Sciences. 3(3): 1-4.

3 Klein M.K. 2006. Tumors of Female Reproductive System. In: Withrow S.J. \& MacEwen E.G. (Eds). Small Animal Clinical Oncology. 4th edn. St. Louis: Saunders Elsevier, pp.610-618.

4 Koushiek Yadav S.P., Rakshith K., Srinivasa Murthy K.M., Kamalakar G. \& Nagaraja B.N. 2019. Surgical management of paravaginal lipoma in a Labrador Retriever bitch - A case report. International Journal of Current Microbiology and Applied Sciences. 8(9): 829-831.

5 Lamagna B., Greco A., Guardascione A., Navas L., Ragozzino M., Paciello O., Brunetti A. \& Meomartino L. 2012. Canine Lipomas Treated with Steroid Injections : Clinical Findings. Public Library of Science. 7(11): 1-5.

6 Laskin W.B., Fetsch J.F., Michal M. \& Miettinen M. 2006. Sclerotic (fibroma-like) lipoma: A distinctive lipoma variant with a predilection for the distal extremities. American Journal of Dermatopathology. 28(4): 308-316.

7 Leil A.Z.A., El-Hallawany H.A., Fadel M.S., Elmesiry A. \& Elsayad A. 2018. Electrosurgical excision and differential pathological diagnosis of external genital tumors in bitch. Alexandria Journal of Veterinary Sciences. 57(2): $1-12$.

8 Manoj K., Dhana L., Sudarshan R. \& Phaneendra M. 2017. A rare case of lipoma of vagina along with perineal hernia in a female dog and surgical treatment. International Journal of Advanced Veterinary Science and Technology. 6(1): 340-345.

9 Nelissen P. \& White R.A.S. 2012. Subtotal vaginectomy for management of extensive vaginal disease in 11 dogs. Veterinary Surgery. 41(4): 495-500.

10 O’Neill D.G., Corah C.H., Church D.B., Brodbelt D.C. \& Rutherford L. 2018. Lipoma in dogs under primary veterinary care in the UK: prevalence and breed associations. Canine Genetics and Epidemiology. 5(9):1-13.

11 Radi Z.A. 2005. Vulvar lipoleiomyoma in a dog. Journal of Veterinary Diagnostic Investigation. 17(1): 89-90.

12 Sontas B.H., Altun E.D., Güvenc K., Arun S.S. \& Ekici H. 2010. Vaginal neurofibroma in a hysterectomized poodle dog. Reproduction in Domestic Animals. 45(6): 1130-1133. 